\title{
Experiencia de cooperación en Turkana (Kenia). Una mirada enfermera.
}

\section{Experience of cooperation in Turkana (Kenya). A nurse look Experiência de cooperação em Turkana (Kenya). Um olhar enfermeira}

Andrés Climent Rubio ${ }^{1}$, José Ramón Martínez Riera ${ }^{2}$, Elena García Higón ${ }^{3}$

${ }^{1}$ Enfermero cooperante en Turkana (Kenia), Vocal de Cooperación Internacional y Relaciones Internacionales de la Asociación de Enfermería Comunitaria (AEC). Enfermero servicio de emergencias Ayuntamiento Teulada-Moraira (Alicante). asandres9@hotmail.com

${ }^{2}$ Enfermero. Presidente Asociación Enfermería Comunitaria (AEC). Profesor Titular Departamento Enfermería Comunitaria, Medicina Preventiva y Salud Pública e Historia de la Ciencia. Facultad Ciencias de la Salud. Universidad de Alicante.jr.martinez@ua.es

${ }^{3}$ Enfermera cooperante en Turkana (Kenia).egarciahigon@gmail.com

Cómo citar este artículo en edición digital: Climent Rubio, A., Martínez Riera, J.R. y García Higón, E. (2015). Experiencia de cooperación en Turkana (Kenia). Una mirada enfermera. Cultura de los Cuidados (Edición digital), 19(43). Disponible en: <http:// dx.doi.org/10.14198/cuid.2015.43.12>

Correspondencia: Servicio de emergencias Ayuntamiento Teulada-Moraira (Alicante).

Correo electrónico: asandres9@hotmail.com

Recibido: 11/03//2015; Aceptado: 10/08/2015

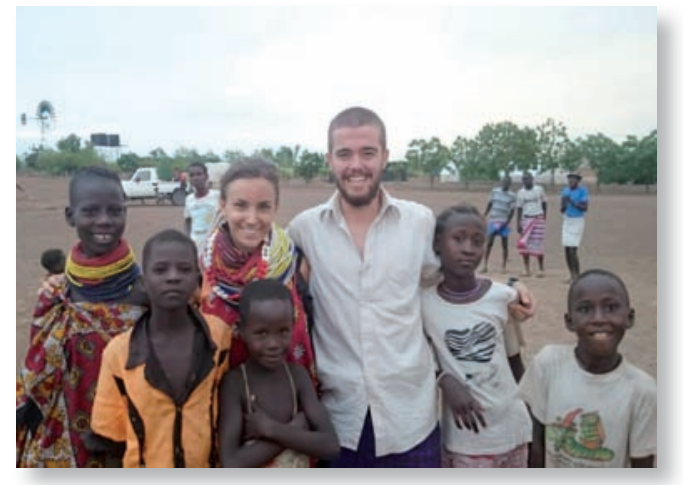

ABSTRACT

Speaking of international cooperation on many occasions we generate some associated with concepts far removed from what really is image.

What we will capture in this experience it is not intended to alarm or create scenarios manipulated or magnify poverty, lack of resources or human misery ... only pretend to be faithful to reality as far and near at the same time as we tell .

Two nurses and a professor at the University of Alicante (Spain) developed in Turkana (Kenya) a draft Community intervention in which public participation is central to its development. The main objective of the intervention is to identify major health needs and how people can respond to them independently and responsibly. The project is initiated by identifying community leaders through whom are involved in the community to begin the process of health education which responds to the health needs of the latter.

After almost a year of intervention have been achieved key objectives related to hygiene, nutrition and care during pregnancy and childbirth.

Keywords: International Cooperation, Community Intervention, Community Participation, Community Nursing.

\section{RESUMO}

Falando da cooperação internacional em muitas ocasiões que gerar algum associado com conceitos muito longe do que realmente é a imagem.

O que vamos capturar nesta experiência que não se destina a criar cenários de alarme 
ou manipuladas ou ampliar a pobreza, a falta de recursos ou a miséria humana ... só que fingir ser fiel à realidade como longe e de perto, ao mesmo tempo que nós dizemos.

Duas enfermeiras e um professor da Universidade de Alicante (Espanha) desenvolvido em Turkana (Kenya) um projecto de intervenção comunitária em que a participação pública é fundamental para o seu desenvolvimento. $\mathrm{O}$ principal objetivo da intervenção é identificar as principais necessidades de saúde e como as pessoas podem responder a eles de forma independente e responsável. O projeto é iniciado através da identificação de líderes comunitários através do qual estão envolvidos na comunidade para iniciar o processo de educação em saúde que responde às necessidades de saúde deste último.

Depois de quase um ano de intervenção foram alcançados objectivos fundamentais relacionados à higiene, nutrição e cuidados durante a gravidez eo parto.

Palavras-chave: Cooperação Internacional, Intervenção Comunitária, Participação Comunitária, Enfermagem Comunitária.

\section{RESUMEN}

Hablar de Cooperación internacional en muchas ocasiones nos genera cierta imagen asociada con conceptos muy alejados de lo que realmente es.

Lo que vamos a plasmar en esta experiencia no pretende alarmar, ni crear escenarios manipulados, ni magnificar la pobreza, la falta de recursos o la miseria humana... tan solo pretende ser fiel a una realidad tan lejana y cercana al mismo tiempo como la que narramos.

Dos enfermeros y un profesor de la Universidad de Alicante (España) desarrollan en Turkana (Kenia) un proyecto de intervención
Comunitaria en la que la participación de la población es el eje de su desarrollo. El principal objetivo de la intervención es la identificación de las principales necesidades de salud y la manera como la población puede responder a ellas de manera autónoma y responsable. El proyecto se inicia con la identificación de los líderes comunitarios a través de los cuales se interviene en la comunidad para iniciar el proceso de educación para la salud que de respuesta a las necesidades de salud de ésta.

Tras casi un año de intervención se han logrado objetivos clave relacionados con la higiene, la alimentación y la atención al embarazo y parto.

Palabras clave: Cooperación Internacional, Intervención Comunitaria, Participación Comunitaria, Enfermería Comunitaria.

\section{Cooperación, una mirada enfermera}

Hablar de Cooperación internacional en muchas ocasiones nos genera cierta imagen asociada con aventura, exploración de algo desconocido, ayuda pero sin saber bien qué tipo, idealismo, solidaridad puntual e incluso cierto morbo por acercarse a la penuria humana (2010, Delgado Caicedo, J. Barrera Castro, A.) (2004, Mazarrasa L, Montero M.J.).

También suele asociarse con proyectos internacionales de grandes ONGs con gran experiencia y muy organizadas.

Pero la Cooperación va mucho más allá de estos sentimientos, pensamientos o imaginaciones asociados a la ignorancia de lo que realmente significa, comporta y aporta. Y más allá, por supuesto de las intervenciones realizadas desde grandes proyectos, que siendo muy importantes, no representan la mayoría invisible de la Cooperación que se realiza (2009, de Almeida Souza, A.). 
Por lo tanto resulta necesario que se conozcan con detalle experiencias, en las que lo vivido va mucho más allá de cualquier imagen previa que nos hubiésemos o nos hubiesen trasladado. Que logren trasladar a la gran mayoría de quienes habitualmente nos situamos como meros espectadores de lo que se hace, vive y sufre de manera muy superflua, tamizada e incluso interesada a través de unos medios de comunicación interesados, mayoritariamente, en otras cuestiones más prosaicas, salvo catástrofe humanitaria que sirva para disparar sus cuotas de audiencia (2008, Casabona Martínez, I. Lillo Crespo, M. Mora Antón, MD. Cort Casabona, S.), (2012, Álvarez S.), (2010, Tassara C.).

Lo que vamos a plasmar en esta experiencia no pretende alarmar, ni crear escenarios manipulados, ni magnificar la pobreza, la falta de recursos o la miseria humana... tan solo pretende ser fiel a una realidad tan lejana $y$ cercana al mismo tiempo como la que narramos. No es nuestra intención tampoco generar lástima o falsa solidaridad. Creemos que es necesario que, huyendo de dramatismos, de eufemismos y de dogmatismos, sepamos contar con la realidad que merece lo que sucede, cómo sucede y dónde sucede y, además, que se haga desde la mirada enfermera de quienes estamos siendo actores voluntarios de esta experiencia de cooperación, enfermeros. Porque, sin duda, la mirada enfermera es intemporal en el sentido de que tiene su origen en la posibilidad humana de autocuidarse y se consolida en "mirada enfermera" en el momento en que se trasciende el instinto y se convierte en servicio. (2003, Alberdi, R.).

\section{África - Kenia - Turkana. La importancia del contexto.}

Para poder hacerse una idea real de la experiencia que vamos a compartir es funda- mental que conozcamos en contexto en el que se desarrolla (2007, Valtueña J.).

Kenia, con 39 millones de habitantes, tiene 47 distritos, cada uno de ellos con un gobierno semiautonómo respecto al gobierno central de Nairobi. La economía de Kenia es además la de mayor producto interno bruto de la región Este y Central de África, lo que resulta paradójico con la realidad de la región donde nos encontramos, el Distrito de Turkana. El Distrito de Turkana se encuentra al noroeste de Kenia en la región del Rift Valley, cuya capital es Lodwar. Su población, según el último censo de 2009, está contabilizada en 850.000 personas repartidas en los $77.000 \mathrm{~km} 2$ que abarca, incluyendo 95.000 refugiados ubicados en el campo de refugiados de Kakuma. El crecimiento anual es del 2,5\%. Hay 92 hombres por cada 100 mujeres, siendo la densidad de población de 11 habitantes por Km2. Pertenecen en su gran mayoría a la etnia Turkana, y su lengua es el Turkana.

La población Turkana se encuentra en una situación de pobreza extrema. Su economía es sumamente frágil, y las sequías periódicas son seguidas de hambrunas y muerte de los sectores de población más débiles -niños y ancianos-. Esta situación determina una esperanza de vida inferior a los 50 años sin contar la mortalidad infantil, que se sitúa en niños menores de 5 años en 170/1000 habitantes. El hambre es el mayor problema que afecta a los Turkana, junto con el aislamiento y la ausencia casi total de todo tipo de infraestructuras, desde carreteras a agua potable, sistema sanitario y medios de transporte.

Mayoritariamente, los Turkana son una etnia de pastores nómadas. Subsisten mediante el pastoreo nómada de cabras, camellos, burros y vacas. Se visten de sus pieles y se alimentan de su leche, sangre y carne, siendo utilizados, 
además, como medio de trueque. Los animales determinan la riqueza y la posición social y condicionan su modo de vida, ya que se desplazan por el distrito en función del acceso a pastos y agua a lo largo del año. Así, durante la época seca habitan las montañas, y en la época de lluvia se desplazan a las planicies.

La mayoría del pueblo Turkana mantiene su religión tradicional monoteísta. Su Dios (AKuj), está asociado con el cielo y es el creador de todas las cosas. A pesar de ello no tiene apenas influencia en sus vidas, y sólo se recurre a él en tiempos de necesidad. Existen médicosadivinos y brujas, cuyas funciones más importantes son predecir la victoria en las guerras, ayudar en la salud y remediar la esterilidad.

La diferenciación entre sexos es muy importante, y diferencian de manera muy clara las funciones de mujeres y hombres. Las mujeres se ocupan de las labores del hogar, cuidado de los hijos, recogida de agua desde los pozos, construcción de la vivienda en caso de nómadas y la mayoría de las tareas que se puedan llevar a cabo dentro de la unidad familiar. Los hombres son los encargados de ordenar las funciones de la mujer y los hijos y el pastoreo de la parte de ganado de la cual no se ocupan sus hijos varones.

A nivel educativo, las niñas y jóvenes se ven marginadas frente a los niños. La oposición a que acudan a la escuela primaria y secundaria parte de sus propias familias, para que así puedan ser casadas a una edad temprana. Solamente en los grandes núcleos de población las mujeres comienzan a desarrollar sus derechos sociales.

\section{Sistema de salud en Turkana}

El sistema de salud, es muy precario. Las enfermedades más frecuentes son la malaria, las enfermedades respiratorias y las infeccio- nes gastrointestinales. Se añaden otras enfermedades endémicas como la brucelosis, el quiste hidatídico o la madurelosis, así como la tuberculosis y el HIV/SIDA, sobre el cual no existen estadísticas fiables en Turkana, aunque supone ya el 33\% de la ocupación de las camas hospitalarias del hospital de Lodwar.

En todo el Distrito Turkana las infraestructuras sanitarias son mínimas. Existen solamente 990 camas hospitalarias repartidas en 5 hospitales, y 81 dispensarios y centros de salud, aunque es más que optimista pensar que todos ellos se encuentren en funcionamiento. La proporción personal sanitario/habitantes es de 1/80.000 en el mejor de los casos. Por lo tanto, el mayor problema de salud es la posibilidad real de acceso por parte de la población a algún centro de salud. Como reflejan las estadísticas gubernamentales publicadas en el Plan de Desarrollo del Distrito Turkana 2002-2008, la distancia promedio al lugar de atención sanitaria más cercano es de $50 \mathrm{Km}$. El hospital principal se encuentra en Lodwar, donde han florecido un buen número de consultas privadas, debido a los limitados servicios del hospital del gobierno.

La atención sanitaria por parte del Ministerio de Salud de Kenia en Turkana se limita a la atención de las poblaciones urbanas del distrito a través de dispensarios. Teniendo en cuenta que aproximadamente el $86 \%$ de la población en Turkana vive en el ámbito rural, la atención sanitaria de facto por parte del gobierno en Turkana es muy limitada. El resto, es llevado a cabo por la Diócesis Católica de Lodwar y por ONG's. Esta labor de suplencia en la atención sanitaria está plenamente aceptada por el Ministerio de Salud, el cual se coordina con las unidades de salud de la Diócesis de Lodwar para campañas en situaciones de epidemias o cuando equipos de especialis- 
tas visitan la zona (desde 2003 grupos de oftalmólogos españoles y desde 2004, grupos de cirujanos acuden a la zona cada año a operar durante 15 días). Asimismo, los dispensarios y centros de salud regentados por la Iglesia Católica informan mensualmente al Hospital central situado en Lodwar, de sus actividades y les remiten estadísticas sanitarias y epidemiológicas. Los dispensarios más alejados de la capital puestos en marcha por el gobierno y situados en los lugares más remotos se encuentran frecuentemente con la desaparición de los profesionales por periodos muy largos de tiempo sin dar respuesta a las necesidades del lugar. Para entender esta visión de la sanidad basta con leer los protocolos hospitalarios en los que se define una emergencia como una situación que puede esperar más de $8 \mathrm{~h}$, Urgencia como situación demorable a más de $24 \mathrm{~h}$ y enfermedad común como atención demorable a más de 7 días. Esto hace que se comprenda un poco mejor la visión de la enfermedad y de su gravedad (2010, Jerez C, García E, Saz M, Tamame M.), (2010, Serra R.)

También es importante describir los niveles de atención que existen en Kenia, que se pueden resumir en los siguientes niveles:

Nivel 1-Atención comunitaria y Comunity Health Worker (CHW)

Nivel 2- Dispensarios. Personal: 1 o 2 enfermeros uno de ellos es el gerente y 102 auxiliares.

Suelen encontrarse en lugares remotos con población muy dispersa y se asientan en poblados seminómadas estables.

Nivel 3- Centros de salud. Personal: 1 Clinical Officer (CO) con función asistencial y además es el gerente del centro, 1 o 2 Enfermeros, 1 o 2 auxiliares, 1 nutricionista, 1 trabajador social. Se encuentran en zonas más importantes con una población estable de envergadura (5000 habitantes)

Nivel 4- Hospitales primarios. Personal: Dependiendo de las dimensiones pero siempre 1 médico y varios $\mathrm{CO}$ o Enfermeros. El médico es el gerente.

Nivel 5- Hospital de referencia. Personal: Hospital que cuenta con unidades de cuidados especiales y con especialistas en diversas áreas médicas.

\section{Las enfermeras en Kenia. Compleja realidad.}

Dentro del sistema de salud de Kenia existen como ya hemos dicho Médicos, Clinical Officer y Enfermeros. La figura del médico queda relegada a grandes hospitales de nivel 5 y en escasas ocasiones a hospitales de nivel 4 llevando a cabo las funciones propias de un médico tal y como las conocemos en España. Los Clinical Officer se sitúan entre el enfermero y el médico y realiza actividades enfermeras y otras propias de la medicina. Podría ser descrito como un enfermero de práctica avanzada con capacidad diagnóstica y de prescripción.

Las Enfermeras, pueden ser generalistas y especialistas (Pediatria, UCI, Anestesia, Quirófano, Salud Mental, Familiar y Comunitaria y Matronas). Dependiendo de donde desempeñen su profesión las enfermeras, con independencia de ser especialistas o no, tienen más o menos competencias. La diferencia entre una enfermera general en Nairobi (capital del país) y una enfermera en Turkana, se centra en que la primera tiene funciones muy parecidas a las de una enfermera española, mientras que la enfermera rural como en Turkana tiene todo tipo de competencias llevando a cabo desde los cuidados más básicos hasta manejar el diagnóstico y tratamiento médico de enfermedades como la tuber- 
culosis (2011, Manjavacas J.), (2003, Palacios García A.L.).

La figura del clinical officer inexistente en nuestro país, no la conoemos dado que trabajan en áreas muy específicas y generalmente en grandes hospitales o grandes núcleos urbanos.

Dentro de esta compleja organización, es donde nos encontramos dos enfermeros españoles formando parte del equipo de salud de la diócesis de Lodwar prestando apoyo a los servicios sanitarios del gobierno, los cuales no son capaces de hacerse cargo de las demandas de salud de la zona donde trabajamos

\section{Las enfermeras en Turkana. Dura realidad.}

Con una visión ya más cercana al contexto en el que trabajamos, estamos en disposición de exponer la de un Enfermero de cooperación internacional en Kenia.

El trabajo en el Centro de Salud no dista mucho de la que se puede llevar a cabo en un Centro de Salud rural en España, con el único matiz de que el enfermero es el único profesional existente para prestar todo tipo de atención sanitaria. En base a ello, atiende la consulta de pacientes agudos (la mayoría de los usuarios); pacientes crónicos con patologías como epilepsia, tuberculosis, VIH; atención a la mujer embarazada; revisión del niño sano e inmunizaciones y todas las urgencias y emergencias que se generan tanto en el poblado como en toda nuestra área de actuación.

Otro aspecto a tener en cuenta es la gran carga de trabajo relacionada con la gestión y administración de recursos. Los recursos de los que se dispone son muy limitados y las posibilidades de obtener repuestos son complicadas o caras. Somos el único punto del proyecto que cuenta con vehículo propio para viajar a la capital donde se nos suministran los recursos. Los desplazamientos son muy costosos en carburante y tiempo dadas las pésimas condiciones de las infraestructuras viarias. El presupuesto, además, es muy limitado puesto que las subvenciones provienen exclusivamente de donaciones particulares y alguna subvención institucional.

Así pues, este fue el contexto y las competencias a las que nos enfrentábamos cuando llegamos. Hay que tener en cuenta que antes de nuestra llegada ya se estaba atendiendo a la población por parte de dos enfermeros españoles que trabajaron en el lugar durante dos años e iniciaron el proyecto. Sin embargo nuestra ilusión era poder desarrollar un proyecto amplio de intervención comunitaria más allá de la atención a la demanda y a la enfermedad que se venía realizando.

\section{Contextualización}

Antes de viajar por primera vez a Turkana el enfermero que iba a sustituir a los dos que llevaban dos años trabajando, autor 1, planteó al Profesor de la Universidad de Alicante Autor 2, la posibilidad de desarrollar un proyecto de intervención como los que había tenido ocasión de conocer en el transcurso de las clases de Intervención Comunitaria. El desconocimiento del contexto y de la situación real del lugar hicieron que en un primer momento se decidiera por parte de ambos planificar exclusivamente la labor de observación y contextualización. Para ello se empleó el primer mes de estancia en Turkana que sirvió también de adaptación. Durante este primer contacto se hizo un análisis del contexto, de la realidad cultural y social del mismo, se identificaron los líderes comunitarios y se valoraron comportamientos y actitudes de las personas a las que se debía atender.

Tras este primer mes, ya de vuelta en España, se llevó a cabo un análisis de la situación 
en base a los datos aportados y se empezaron a planificar los objetivos y actividades que se querían llevar a cabo cuando regresase para incorporarse definitivamente en Turkana. Inicialmente se plantearon dos ejes básicos de trabajo, el trabajo con parteras tradicionales y con los conocidos como Community Health Workers (CHW).

\section{Inicio de la intervención}

De nuevo en Turkana, convivimos los dos equipos de salud durante unos meses, los que iniciaron el proyecto y los que íbamos a reemplazarles. Tras plantear la idea que llevábamos planificada desde España de desarrollar una Intervención Comunitaria en la que se incorporase la participación activa de la población atendida, la idea no acabó de ser entendida ni asumida, por lo que se prefirió paralizar su puesta en marcha hasta que se produjese el relevo definitivo. Durante el tiempo en que permanecimos los dos equipos optamos por continuar con la importante labor de observación y de recogida de información que posteriormente fue de gran valor para el desarrollo del proyecto. Además nos encontramos con un nuevo inconveniente que hacía oportuno el retraso de su puesta en marcha y fue la ausencia temporal de la líder comunitaria identificada en la primera visita.

Con la incorporación de la nueva enfermera, Autor 3, y la marcha definitiva del anterior equipo empezamos a trabajar.

El primer paso fue reunir al grupo de las matronas tradicionales y convencerlas de la oportunidad de las reuniones ya que el equipo había cambiado y teníamos cosas nuevas que plantear. Una vez convencidas de retomar las sesiones pidieron como es costumbre aquí una gratificación económica por asistir a las reuniones. Esta situación en la que la población pide dinero o comida por asistir a reuniones o cursos en los que se les da formación suele ser su único beneficio y sigue siendo práctica habitual por parte de otras ONGs. Nuestro objetivo fue el de eliminar la dependencia y cambiar la mentalidad de que por cada esfuerzo que se hiciese tuviesen que recibir una gratificación económica aunque fuese en su propio beneficio. Esto se logró de manera muy gradual gracias a la ayuda de la líder que a su vez interactúa como traductora (2007, Carrera JM, Devesa N, Chacón D, Cararach V, Fabre E, Foradada C, Miguel J, Prats P, Rubio R.).

El siguiente paso fue con los CHW. Aunque el planteamiento inicial tuvo que ser modificado por ser excesivamente ambicioso. Por ello, tuvimos que priorizar, y se decidió empezar trabajando en la comunidad semi-nomada y estable que se encuentra dentro de nuestro área de trabajo. Para seguir avanzando tuvimos que identificar más líderes comunitarios, tarea nada sencilla, dado que estos debían reunir cualidades muy poco frecuentes en Turkana, como son hablar inglés o swahili, tener interés por la salud no solo propia sino de los demás, no pedir gratificación económica y ser un referente para el resto del pueblo. Finalmente identificamos al segundo líder, que incorporamos como miembro del equipo de salud, como traductor y como líder comunitario, es decir como Community Health Worker. Identificados ambos líderes se empezó a trabajar con ellos dos aspectos básicos, primeros auxilios e higiene básica.

Consideramos también trabajar con los maestros de la escuela primaria y de infantil. Contactamos con ambos y se habló con ellos de higiene y de cómo incorporarla en los juegos infantiles. Aquí se planteó el objetivo inicial de conseguir una ducha semanal con agua 
y jabón del cuerpo entero y un lavado de manos y cara diario antes de las dos comidas que reciben.

Con estos tres objetivos de trabajo nuestra presencia en las escuelas se consolidó semana tras semana. Se identificaron dos $\mathrm{CHW}$ más y el grupo de matronas aumentó dado que pedimos que cada una de las anteriores trajese a otra que sería su "alumna”.

Los avances empezaron a ser claramente visibles, en el grupo de matronas. Con una buena base sobre la higiene personal se logra cambiar un aspecto del post parto con mucha tradición y que genera mucho miedo como es el tocar al niño justo después del parto. Por tanto, podemos decir que conseguimos introducir el método canguro dentro de las matronas tradicionales en relativamente poco tiempo. Por otro lado el grupo de $\mathrm{CHW}$ empieza a adquirir habilidades en promoción de la salud y prevención de la enfermedad, ya que no solo son capaces de adquirirlas para ellos mismos sino que son ellos mismos los que toman la iniciativa de trasladar sus conocimientos al resto de la población, convirtiéndose en verdaderos promotores de salud.

Día a día se observan cambios dentro de la comunidad. Aunque el cambio de mentalidad que identifique el trabajo en/con la comunidad sin recibir compensación económica o en especias, aún queda lejos, se empieza a ver un cambio real. Pequeños gestos como el crear un grupo de trabajo para construir la valla del centro de salud a cambio de comida, la cual procede del gobierno y a priori debería ser repartida sin exigirles nada a cambio empiezan a ser una realidad. También en la escuela primaria los niños empiezan a no temer al agua $y$ a disfrutar con la ducha semanal. Por su parte el maestro se muestra muy motivado y ya de forma autónoma toma la iniciativa de llevar a cabo las actividades de higiene básica con los niños.

Todos estos avances son compartidos, analizados y evaluados por nosotros mismos y con el Profesor Autor 2 en España con el que mantenemos una permanente comunicación. Lo logrado nos anima y permite plantear un nuevo reto en la Intervención como fue el organizar una reunión con los miembros más representativos del poblado para llevar a cabo un grupo nominal que nos permitiese identificar necesidades sentidas por parte de la comunidad. La reunión en si fue muy útil aunque no acudieron quienes inicialmente habíamos previsto. En un primer lugar nos encontramos con los CHW y el maestro de infantil pero unos minutos más tarde empezaron a sumarse mujeres del pueblo. Con ello nos dimos cuenta de que el planteamiento para el grupo nominal lo habíamos realizado en base a premisas falsas al no identificar y valorar la diferencia conceptual que tienen en torno a la salud y la nula percepción en relación a la toma de decisiones. Esto nos hizo replantearnos los objetivos y la dinámica grupal a seguir que no a abandonar el objetivo de realizarla.

Semanas después conseguimos la ansiada reunión con los líderes políticos y las personas más representativas de la comunidad. De ella salieron grandes ideas y nuevos objetivos. Nos expresaron sus principales necesidades y aspectos que a su modo de ver les podrían ayudar en la prevención de la enfermedad. Las principales preocupaciones identificadas fueron:

- La malaria.

- La falta de agua.

- La falta de letrinas.

- La diarrea.

Cada punto fue analizado individualmente y de manera participativa. Del análisis rea- 
lizado se concluyó llevar a cabo dos nuevas intervenciones: reactivar las relaciones con la comunidad misionera para conseguir agua $y$ activar el trabajo con los CHW sobre la prevención de la diarrea.

Las intervenciones mencionadas anteriormente se concretaron finalmente en los siguientes dos objetivos: Conseguir muertes por malaria 0 y obtener una letrina por familia. A lograr a través del desarrollo de un programa de actuación estructurada y participativa.

Dentro del programa de la malaria se contempla conseguir mosquiteras a bajo precio para poder ser vendidas a la población y lograr de este modo que el mayor numero de habitantes posea una que evite las picaduras (2010, Lanaspa M., Renom M., Bassat Q.), (2004, Alonso, PL. Guinovart, C.), (2006, Sochantha, T. et al.)

Por su parte con el grupo de matronas y en la atención al embarazo en el centro de salud se plantea que toda mujer seguida por nuestro equipo durante el embarazo recibirá una mosquitera a mitad de precio y si el parto es asistido por el equipo esta no tendrá coste alguno para la mujer. Esta medida tiene una doble finalidad para las embarazadas, que puedan acceder a una mosquitera y que sean seguidas en el embarazo y atendidas en el parto.

Por último en la prevención de muertes por malaria se empezó a trabajar con los CHW y toda persona capaz de comprender como realizar el test, para que pudieran realizarlo de manera autónoma, dado que a través de la incorporación del uso de pruebas rápidas por promotores de salud y de estrategias educativas se mejora la oportunidad en el diagnóstico y tratamiento apropiado de la malaria (2013, Mendoza Nohora, M. Cucunubá Zulma, M. Aponte Samanda, N. González, E. Bernal Sindy D.), (2008, Casapia, Mar- tín et al.), (2006, Alvarado Beatriz, E. Gómez Elizabeth, M. Serra, Carvajal R. Carrasquilla, G.).

Por lo que se refiere al programa de las letrinas, el objetivo es dotar de una letrina a cada familia. El coste de crear una letrina tal y como se conceden es alto pero con una nueva técnica que uno de nuestros voluntarios conoce el coste se reduce significativamente y las ventajas se multiplican (2013, Santamaría Rodriguez, L.).

En estos momentos nos encontramos en la recopilación de datos sobre todas estas intervenciones descritas para poder realizar las evaluaciones que permitan identificar la eficacia y eficiencia de las mismas.

De todo lo trabajado podemos concluir que:

- La Cooperación internacional no puede basarse exclusivamente en la atención a la demanda y a la enfermedad ya que genera dependencia y no favorece el cambio de hábitos y conductas que se identifican como nocivas para la salud de la población atendida.

- Es fundamental conocer el contexto de actuación, en el que deben identificarse la cultura, historia, normas, valores, creencias... antes de llevar a cabo ningún tipo de intervención.

- La identificación de líderes comunitarios y de agentes de salud que se incorporen como verdaderos agentes promotores de salud es imprescindible para iniciar cualquier proyecto de intervención comunitaria que pretenda el acceso a la población y la incorporación/modificación de hábitos de conducta relacionados con la salud.

- Las enfermeras están en disposición y tienen las competencias necesarias para ofrecer una adecuada atención centrada en la par- 
ticipación comunitaria que permita identificar las necesidades sentidas de la población e iniciar el trabajo compartido para lograr la autonomía individual y colectiva suficientes para satisfacer las demandas generadas.

- Las intervenciones basadas en la participación de la población atendida son identificadas como positivas e incorporadas de manera mucho más activa y temprana por sus habitantes.

- Los costes de las intervenciones son muy bajos y los resultados que se obtienen representan una garantía de futuro en la promoción de la salud y la prevención de la enfermedad.

\section{Bibliografía}

- Alberdi Castell RM, Cuxart Ainaud N. (2005) Cuidados, enfermeras y desarrollo profesional: Una reflexión sobre las bases del ejercicio profesional. Presencia jul-dic;1(2). Recuperado de http://www.index-f.com/presencia/ n2/23articulo.php

- Alonso, P.L. Guinovart, C. (2004). Malaria: situación, instrumentos de control, recursos y soluciones. Ars Medica. Revista de Humanidades, 2:166-179.

- Alvarado Beatriz, E. y Gómez E. Serra, M. y Carvajal, R. y Carrasquilla, G. (2006). Evaluación de una estrategia educativa en malaria aplicada en localidades rurales del Pacífico colombiano. Biomédica [serie en Internet]: 26 (3): 342-352. Recuperado de: http://www. scielo.org.co/scielo.php?script=sci_arttext\&pid=S0120$41572006000300004 \& \ln \mathrm{l}=\mathrm{en}$

- Álvarez S. (2012). Una introducción a la Cooperación Internacional al Desarrollo. Redur, 10, 285-309.

- Carrera J.M. y Devesa N y Chacón D y Cararach V y Fabre E y Foradada C y Miguel J y Prats P y Rubio R. (2007). Mortalidad materna en África. Prog Obstet Ginecol, 50(7): 407-19.

- Casabona Martínez, I. Lillo Crespo, M. Mora Antón, MD. Cort Casabona, S. (2008). Visión de la enfermería en Ruanda, relato de una experiencia de docente. Cultura de los cuidados, Año XII (24): 82-7.

- Casapia, Martín et al. (2008). Mejora en el diagnóstico y tratamiento oportuno de malaria con el uso de pruebas rápidas por promotores de salud en la Amazonía peruana. Rev. perú. med. exp. salud pública, Lima, 25(4). Recuperado de: http://www.scielo.org.pe/scielo.php?script=sci_ arttext\&pid $=$ S172646342008000400003\&lo

- De Almeida Souza, A. (2009). Enfermería en el discurso politico internacional. Rev Adm Sanit, 7(2):191-206.

- Delgado Caicedo, J. y Barrera Castro, A. (2010). Subiendo al Sur: África y América Latina en las nuevas dinámicas de la cooperación Sur. Humania del Sur, 5 (8): 89-106.

- Jerez C y García E y Saz M y Tamame M.(2010). Colaborar con África: la experiencia del retorno. Index de Enfermería, 19(2-3): 111-114.

- Lanaspa M. y Renom M. y Bassat Q. (2010). La malaria en el mundo en 2010: ¿qué hay de nuevo acerca de esta vieja enfermedad? Rev Pediatr Aten Primaria [revista en la Internet]: 12(48): 685-700. Recuperado de: http://scielo.isciii.es/scielo.php?script=sci_arttext\&pid=S113976322010000600013\&lng=es

- Manjavacas J. Presencia enfermera en la acción humanitaria. Documentos Enfermería. 2011; (41):17-23.

- Mazarrasa L y Montero M.J. (2004). Cooperación internacional española en el ámbito de la salud. Gac Sanit, 18(Supl 1):214-20

- Mendoza Nohora M y Cucunubá Zulma M y Aponte Samanda, E y González Bernal S. (2013). Evaluación de campo de la Precisión de la prueba de Diagnóstico Rápido $S D$ Bioline Malaria Antígeno Pf / $P V^{*}$ en Colombia.Biomédica [serie en Internet]: 33 (4): 587-597. Recuperado de: http://www.scielo.org.co/scielo.php?script=sci_ar ttext\&pid=S012041572013000400012\&lng=en.http:// dx.doi.org/10.7705/biomedica.v33i4.1464.

- Palacios García AL. (2003) Las Enfermeras y el voluntariado. Opinión y participación de profesionales de enfermería en ONG. Index de Enfermería [Index Enferm] (edición digital): 40-41. Recuperado de: <http://0-www. index-f.com.avalos.ujaen.es/index-

- Santamaría Rodriguez, L. (2013). Proyecto de prevención de las enfermedades diarreicas en la comunidad rural de Kasenga, República Democrática del Congo (Trabajo 


\section{Cultura de las Cuidados}

Fin de Master no publicado). Universidad de Valladolid. Facultad de Filosofía y Letras. Recuperado de: http:// uvadoc.uva.es/handle/10324/4628

- Serra R. (2010). Conocer nos ayuda a comprender. Experiencia en África: estancia en un hospital de Costa de Marfil. Arch Memoria, 7(3) Recuperado de: http://www. indexf.com/memoria/7/3703.php

- Sochantha, T. et al. (2006). Mosquiteros tratados con in- secticida para la prevención de Plasmodium falciparum malaria en Camboya: un ensayo aleatorio grupal. Medicina Tropical y Salud Internacional, 11 (8): 1166-1177.

- Tassara C. (2011). Paradigmas, actores y políticas. Breve historia de la cooperación internacional al desarrollo. Revista UNAULA, 31: 41-97.

- Valtueña J.(2007). Investigación sanitaria en los países pobres. Offarm, 26(2): 27-31.

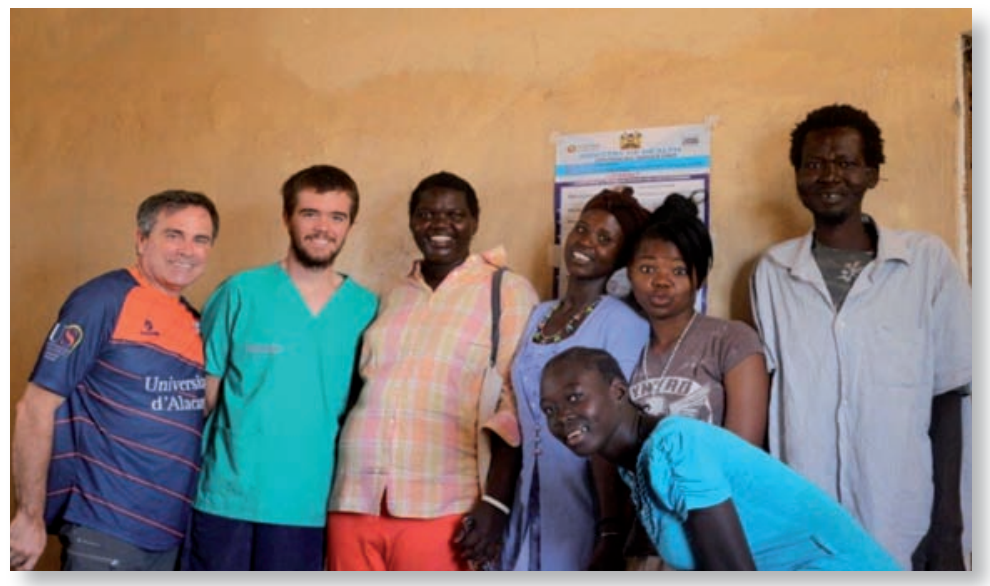

